\title{
Segmentation of Leaf Images Based on the Active Contours
}

\author{
Wu Peng, Li Wenlin and Song Wenlong* \\ College of Mechanical and Electronic Engineering, Northeast Forestry \\ University, \\ Harbin, 150040, China \\ wlsong139@126.com
}

\begin{abstract}
Leaves contain important genetic information which can be used as a basis for the identification of plants. As a first step of modeling virtual three-dimensional plant, how to extract visual characteristic information form leaf images has great significance. We propose an optimized $C$-V model in this paper, which can detect objects in homogeneous regions of given leaf images and speed up running time. The new method combines local information with global information and optimizes the defect that SDF needs to be reconstructed partially so that the energy function is improved. Experimental results show that our algorithm can stop active contours on the correct boundary, get accurate image segmentation, and the speed is more than 1.5 times faster to $C$-V model.
\end{abstract}

Keywords: image segmentation, active contours, $C$-V model, leaf image

\section{Introduction}

Because of the significance of biological and iconography, leaf is an important element of modeling virtual plant [1]. As the first step to three-dimensional reconstruction of plant, leaf image extraction becomes increasingly popular [2-5].

In recent years, because of its outstanding performance in the field of image segmentation, active contour models [5-9] have received more attention in image processing. Defined by the energy function, active contours model can capture the desired boundary by calculating the partial differential equation (PDE). Based on the Osher and Sethian's level set method [10-11] by mean curvature [12] and Mumford-Shah segmentation model [13], Chan and Vese have proposed a new model which could detect object boundary without edges [14] which called C-V model. The C-V model uses mean-curvature flow to evolve active contours. Li Jun et al., have improved C-V model by adding a fast marching method to calculate signed distance function (SDF) [15]. Lee et al., have solved the shortcoming of $\mathrm{C}-\mathrm{V}$ model which may not capture desired contours in weak edges [16]. Brox et al., detect edges with multiple regions based on Mumford-Shah functional [17-19].Chan propose a level set based variational approach that incorporates shape priors into $\mathrm{C}-\mathrm{V}$ model [20].

However, these models are not based on the research of plant organs. For modeling virtual plant, we still need further research on extraction of leaf images. This paper presents an improved $\mathrm{C}-\mathrm{V}$ model for detecting boundary in given leaf images which introduces local statistical information and an internal energy functional item into energy function of classic C-V model. Many experiments have been done and results show our model can accurately 
extract the wanted edges of leaves.

\section{C-V Model}

$\mathrm{C}-\mathrm{V}$ model is an improved active contours model, inspired by Mumford-Shah's partition model and Osher and Sethian's level sets model. Here comes the energy function of active contours $\mathrm{C}-\mathrm{V}$ model:

$$
\begin{gathered}
\mathrm{F}\left(c_{1}, c_{2}, \mathrm{C}\right)=\mu \cdot \operatorname{Length}(\mathrm{C})+v \cdot \text { Area(inside }(\mathrm{C})+\lambda_{1} \int_{\text {inside }(C)}\left|u_{0}(x, y)-c_{1}\right|^{2} d x d y+ \\
\lambda_{2} \int_{\text {outside }(C)}\left|u_{0}(x, y)-c_{2}\right|^{2} d x d y
\end{gathered}
$$

where $\mathrm{C}$ is the variable evolving curve, and defined by $\mathrm{C}$, the constants $c_{1}$ and $c_{2}$ are parameters of image $u_{0}$ inside $\mathrm{C}$ and outside $\mathrm{C}$, relatively. Length $(\mathrm{C})$ and Area (inside $(C)$ ) are constraints for the function. Last two items are fitting items of the energy function, they obtain the minimum value when active contours on the boundaries. Parameters $\mu>0, v \geq 0, \lambda_{1}, \lambda_{2}>0$ are fixed. The problem of $\mathrm{C}-\mathrm{V}$ active model turns into minimizing the energy function $F\left(c_{1}, c_{2}, C\right)$, i.e. $\inf \left(c_{1}, c_{2}, C\right) F\left(c_{1}, c_{2}, C\right)$.

Replace evolving curve $\mathrm{C}$ with a level set function $\phi$, using the Heaviside function, and the Dirac function:

$$
\begin{gathered}
H(z)=\frac{1}{2}\left(1+\frac{2}{\pi} \arctan \left(\frac{z}{e}\right)\right) \\
\delta_{0}(z)=\frac{d}{d z} H(z)=\frac{\varepsilon}{\pi\left(\varepsilon^{2}+z^{2}\right)}
\end{gathered}
$$

Then, define constraints Length $\{\phi=0\}$ and $\operatorname{Area}\{\phi \geq 0\}$ by energy function:

$$
\begin{gathered}
\text { Length }\{\phi=0\}=\int_{\Omega}|\nabla H(\phi(\mathrm{x}, \mathrm{y}))| d x d y=\int_{\Omega} \delta_{0}(\phi(\mathrm{x}, \mathrm{y}))|\nabla \phi(\mathrm{x}, \mathrm{y})| d x d y \\
\text { Area }\{\phi \geq 0\}=\int_{\Omega} \nabla H(\phi(\mathrm{x}, \mathrm{y})) d x d y
\end{gathered}
$$

The functions $H$ and $\delta_{0}$ are regularized when $\varepsilon \rightarrow 0$, denoting them as $H_{\varepsilon}$ and $\delta_{\varepsilon}$. Now the regularized energy functional formulation can be written as:

$$
\begin{array}{r}
F_{\varepsilon}\left(c_{1}, c_{2}, \phi\right)= \\
\mu \int_{\Omega} \delta_{\varepsilon}(\phi(x, y))|\nabla \phi(x, y)| d x d y+v \int_{\Omega} H_{\varepsilon}(\phi(x, y)) d x d y+ \\
\lambda_{1} \int_{\Omega}\left|u_{0}(x, y)-c_{1}\right|^{2} H_{\varepsilon}(\phi(x, y)) d x d y+\lambda_{2} \int_{\Omega}\left|u_{0}(x, y)-c_{2}\right|^{2}\left(1-H_{\varepsilon}(\phi(x, y))\right) d x d y \quad(6)
\end{array}
$$

Minimizing the function $F_{\varepsilon}\left(c_{1}, c_{2}, \phi\right)$ as $\phi$ fixed to get $c_{1}(\phi)$ and $c_{2}(\phi)$. According to variational method, the associated Euler-Lagrange equation can be deduced to minimize $\mathrm{F}_{\varepsilon}$ when keeping $c_{1}(\phi)$ and $c_{2}(\phi)$ fixed. Based on the steepest descend method [21], and the descent direction is defined by a time parameter $t \geq 0$, the level sets evolving function becomes:

$$
\left\{\begin{array}{c}
\frac{\partial \phi}{\partial t}=\delta_{\varepsilon}(\phi)\left[\mu \operatorname{div}\left(\frac{\nabla \phi}{|\nabla \phi|}\right)-v-\lambda_{1}\left(u_{0}-c_{1}\right)^{2}+\lambda_{2}\left(u_{0}-c_{2}\right)^{2}\right] \\
\mathrm{c}_{1}(\phi)=\frac{\int_{\Omega} u_{0}(x, y) H(\phi(\mathrm{x}, \mathrm{y})) d x d y}{\int_{\Omega} H(\phi(\mathrm{x}, \mathrm{y})) d x d y} \\
\mathrm{c}_{2}(\phi)=\frac{\int_{\Omega} u_{0}(x, y)(1-H(\phi(\mathrm{x}, \mathrm{y}))) d x d y}{\int_{\Omega}(1-(H(\phi(\mathrm{x}, \mathrm{y}))) d x d y}
\end{array}\right.
$$

It can be learn from those evolving functions that $\mathrm{C}-\mathrm{V}$ model does not stop evolving curves by an edge function; so on detection of smooth boundary, $\mathrm{C}-\mathrm{V}$ model can perform better when classical snakes methods may not applicable, even in noisy images. Due to its significant advantages, $\mathrm{C}-\mathrm{V}$ model in the field of image segmentation has been widely applied.

However, in practice, the shortcoming of C-V model which can't detect correct curves in homogeneous regions where contain the desired edges has been gradually discovered. And in practical application, it's necessary to speed up computing time for extraction and segmentation of a large number of leaf images. 


\section{The Improved C-V Model for Leaf Images Segmentation}

Leaves contain genetic information of plants, and depending on their shapes we can identify plants. Therefore, it is essential to extract desired boundaries accurately and efficiently from leaf images. From above analysis, we need to improve $\mathrm{C}-\mathrm{V}$ model from two aspects. The basis of building organ elements database in the three-dimensional reconstitution project of leaf organs is the collecting and extracting of leaf image. In the growing process, however, plants sometimes have been eaten by insects inevitably. Coupled with virus attacks, there have been some holes existing on leaves occasionally. How to segment those leaf images and getting feature extractions is of great importance in virtual plants modeling. So it's imperative that the improved model should overcome C-V model's shortcoming which cannot segment accurately in inhomogeneous regions of given images. Furthermore, to build different kinds of leaves' database needs to spend a lot of time in image segmentation. It's of great significance to improve the speed of the algorithm especially when processing massive amounts of leaf images.

Based on the above two reasons, we have improved the $\mathrm{C}-\mathrm{V}$ model from two aspects in the sections below.

\subsection{Combining with Local Information}

The stopping term of $\mathrm{C}-\mathrm{V}$ model originates in Mumford-Shah segmentation methods which expressing one image as the following form:

$$
u(x, y)=u^{+} H(\phi(x, y))+u^{-}(1-H(\phi(x, y)))
$$

where $u(x, y)$ is the given image, and $\mathrm{u}^{+}, \mathrm{u}^{+}$, is the region inside the evolving curve and respectively outside the curve. The undetermined coefficients $u^{+}, u^{-}$of the model are depending on the two smoothly partitioned regions of $u(x, y)$, respectively. This segmentation technique is based on gray level decision criterion to divide regions. The $\mathrm{C}-\mathrm{V}$ model stops curve evolution by using a similar idea. If there isn't significance difference of pixels' gray value between the inner region and outer region, and the gray-level distribution of leaf image isn't homogeneous, the active contours using $\mathrm{C}-\mathrm{V}$ model will not stop on correct boundaries. Moreover, it can be seen easily from equation (7) that $\mathrm{C}-\mathrm{V}$ model only takes global information into consideration. The improved $\mathrm{C}-\mathrm{V}$ model combines global and local information in order to get better segmentation results. In the regions of interest, local information acts to improve the adaptability for withered or eaten leaves [22].

We obtain local information $m, n$ by calculating gray scale average values using a $5 \times 5$ mask.

$$
\begin{gathered}
\mathrm{m}(\phi(\mathrm{x}, \mathrm{y}))=\frac{\sum_{i=-2}^{2} \sum_{j=-2}^{2} u_{0}(x+i, y+j) H(\phi(x+i, y+j))}{\sum_{i=-2}^{2} \sum_{j=-2}^{2} H(\phi(x+i, y+j))} \times H(\phi(x, y)) \\
\mathrm{n}(\phi(\mathrm{x}, \mathrm{y}))=\frac{\sum_{i=-2}^{2} \sum_{j=-2}^{2} u_{0}(x+i, y+j)(1-H(\phi(x+i, y+j)))}{\sum_{i=-2}^{2} \sum_{j=-2}^{2}(1-H(\phi(x+i, y+j)))} \times(1-H(\phi(x, y))
\end{gathered}
$$

By acquiring the global information and local contrast information of the current pixel, we can take intensity inhomogeneity which appears in some complex leaf images into consideration. This method provides a viable option for evolving curves to get better segmentation in sophisticated images, such as image of curly leaves. We combine local information with global information then calculate arithmetic mean of them. The energy equation becomes:

$$
\begin{array}{r}
F_{\varepsilon}\left(c_{1}, c_{2}, \phi\right)= \\
\mu \int_{\Omega} \delta_{\varepsilon}(\phi(\mathrm{x}, \mathrm{y}))|\nabla \phi(\mathrm{x}, \mathrm{y})| d x d y+v \int_{\Omega} H_{\varepsilon}(\phi(\mathrm{x}, \mathrm{y})) d x d y+ \\
\lambda_{1} \int_{\Omega}\left|u_{0}(x, y)-\frac{c_{1}+m}{2}\right|^{2} H_{\varepsilon}(\phi(\mathrm{x}, \mathrm{y})) d x d y+\lambda_{2} \int_{\Omega}\left|u_{0}(x, y)-\frac{c_{2}+n}{2}\right|^{2}\left(1-H_{\varepsilon}(\phi(\mathrm{x}, \mathrm{y}))\right) d x d y
\end{array}
$$




\subsection{Internal Energy Functional Term}

The function $\phi$ will be made too much flat because of sale adjustment when it is from initial level sets function to SDF (Signed Distance Function) of zero-level curve through using level sets and Dirac measure $\delta_{0}$. Meanwhile, the evolvement of active contours will be influenced because of the reinitializing of SDF [23-24].

It is considered to add internal energy functional to energy function so that the way of reinitializing of level sets function will be improved. Internal energy functional $F_{\text {in }}(\phi)$ $[25]$ is

$$
F_{\text {in }}(\phi)=\int_{\Omega} \frac{1}{2}(|\nabla \phi|-1)^{2} d x d y
$$

Internal energy functional $F_{\text {in }}(\phi)$ will describe the degree how level set deviates from SDF, reduce deviation of $\phi$ function from SDF by numerical calculation, optimize the need of reinitializing SDF. The new energy function (12) can be worked out by adding (11) and local information of (10) to Internal energy functional.

$$
\begin{array}{r}
\mathrm{F}_{\mathrm{new}}\left(c_{1}, c_{2}, \phi\right)= \\
\mu \int_{\Omega} \delta_{\varepsilon}(\phi(\mathrm{x}, \mathrm{y}))|\nabla \phi(\mathrm{x}, \mathrm{y})| d x d y+v \int_{\Omega} H_{\varepsilon}(\phi(\mathrm{x}, \mathrm{y})) d x d y+ \\
\lambda_{1} \int_{\Omega}\left|u_{0}(x, y)-\frac{c_{1}+m}{2}\right|^{2} H_{\varepsilon}(\phi(\mathrm{x}, \mathrm{y})) d x d y+ \\
\left.\lambda_{2} \int_{\Omega}\left|u_{0}(x, y)-\frac{c_{2}+n}{2}\right|^{2}\left(1-H_{\varepsilon}(\phi(\mathrm{x}, \mathrm{y}))\right) d x d y\right)+\int_{\Omega} \frac{1}{2}(|\nabla \phi|-1)^{2} d x d y
\end{array}
$$

An equation of a new level set evolution can be worked out by solving Euler-Lagrange equation of (12) to get a minimum value, according to steepest descend method and introducing artificial time parameter $\mathrm{t}>0$. The equation is:

$$
\begin{gathered}
{\frac{\partial \phi}{\partial t_{n e w}}}_{\text {ne }}=\delta_{\varepsilon}(\phi)\left[\mu \operatorname{div}\left(\frac{\nabla \phi}{|\nabla \phi|}\right)-v-\lambda_{1}\left(u_{0}-\frac{c_{1}+m}{2}\right)^{2}+\lambda_{2}\left(u_{0}-\frac{c_{2}+n}{2}\right)^{2}\right]+\operatorname{div}[(1- \\
\left.\left.\frac{1}{|\nabla \phi|}\right) \nabla \phi\right]
\end{gathered}
$$

$\mathrm{m}, \mathrm{n}$ are local information constants in the equation (10). $\varepsilon=1.5$ in regularized $\delta_{\varepsilon}(\phi)$. Assuming the image size is $\mathrm{M} \times \mathrm{N}, \quad 0 \leq i \leq M, 0 \leq j \leq N$, and the space step is $\mathrm{h}$, grid points $\left(x_{i}, y_{j}\right)=(i h, j h)$ can be worked out in the matrix of the formation of leaf image. Get the derivative with finite difference in the process of numerical approximation. That is:

$$
\left\{\begin{array}{c}
\frac{\partial \phi}{\partial x}=\phi(i+1, j)-\phi(i, j) \\
\frac{\partial^{2} \phi}{\partial x^{2}}=\phi(i+1)-2 \phi(i, j)+\phi(i-1, j)
\end{array}\right.
$$

As the same way, we can get the PDF about y.

Initializing level set $\phi_{0}$, by $n=0$, and compute $c_{1}, c_{2}, m, n$. The evolution curve of active contours can be got through solving the PDE in $\phi$ from (13) and iterating to obtain $\phi_{i, j}{ }^{\mathrm{n}+1}$.

\section{The Experimental Results}

Based on the analysis above about the experiments of leaf image edge extraction, the mode proposed in this paper should reach the following standards compared with classical $\mathrm{C}-\mathrm{V}$ model.

It can accurately segment the leaf edge image, including the leaves with more holes and incomplete surface.

The calculation speed will be improved compared with C-V model.

In order to verity that the new algorithm is right according to the two standards, the author chose two leaf images of different kinds of trees to test.

In our experiments, the parameters are chosen as follows: $\mu=0.2, \quad, \quad v=0$, $\lambda_{1}, \lambda_{2}=1, \Delta t=1, h=1$. 
Based on the size of the experimental leaf images, set the initial level set function $\phi_{0}(x, y)=-\sqrt{(x-60)^{2}+(y-60)^{2}}+58$. Calculate the running time in seconds of algorithm by the time functions tic and toc in Matlab2012.

Figure 1 is the experimental results of an intact palmate leaf with the image size of $446 \times 1434$. Compare Figure 1(a) with Figure 1(c), we can see our model works better than the $\mathrm{C}-\mathrm{V}$ model on leaf's boundary which can be more clearly identified at gaps between lobes of given leaf: the edge showed in Figure 1(c) seems a litter sharper than in Figure 1(a). Making analysis of the image segmentation results Figure 1(b) and Figure 1(d), due to the high contrast we can see clearly that the improved model gets accurately extraction result.

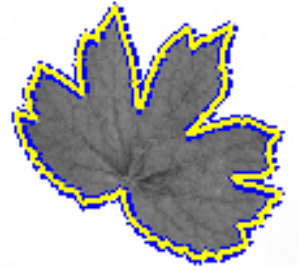

(a) Detection of the C-V model

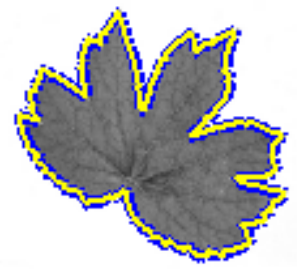

(c) Detection of the improved model

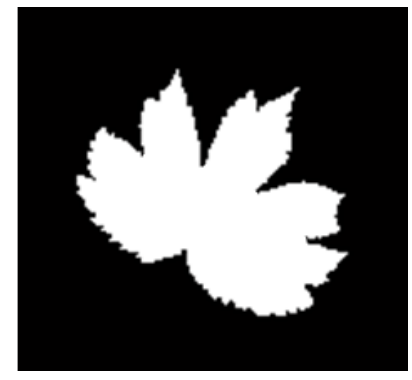

(b) Segmentation of the C-V model

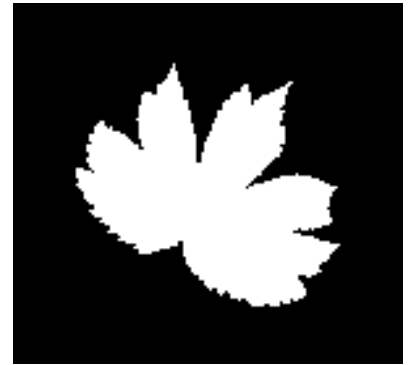

(d) Segmentation of the improved model

Figure 1. Experimental Results of an Intact Palmate Leaf

Figure 2 is the experimental results of a withering leaf with holes, with the image size of $400 \times 2040$. From Figure 2(a) and Figure 2(c), it shows obviously that in Figure 2(c) the active contours stop on the desired boundaries, accurately. First, in Figure 2(a) active contours can't detect the leaf apex; second, there are three adjacent holes separated by two narrow edges on the surface of the withering leaf, and in Figure 2(a) C-V model doesn't obtain accurate leaf margins, but in Figure 2(c) the improved model does. In segmented images Figure 2(b) and Figure 2(d), we can see obviously the improved model is better than the classical C-V model.

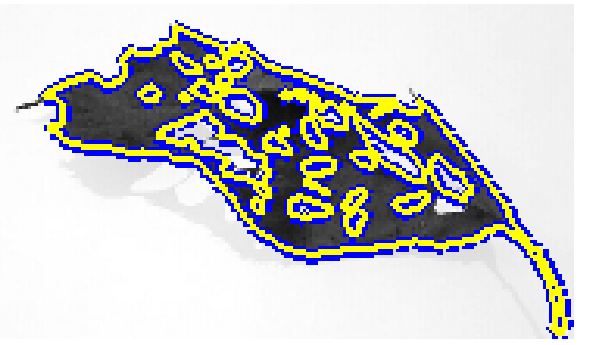

(a) Detection of the C-V model

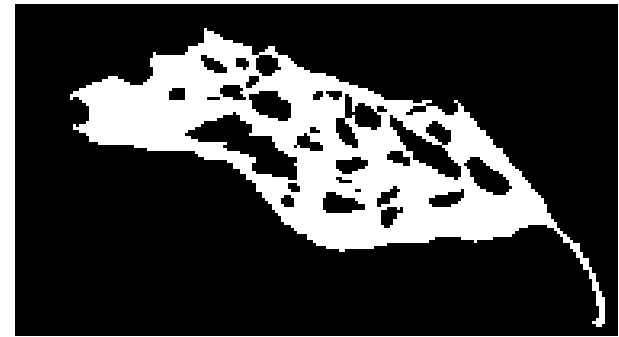

(b) Segmentation of the C-V model 


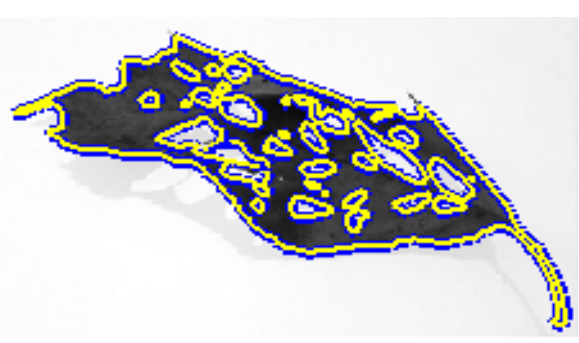

(c) Detection of the improved model

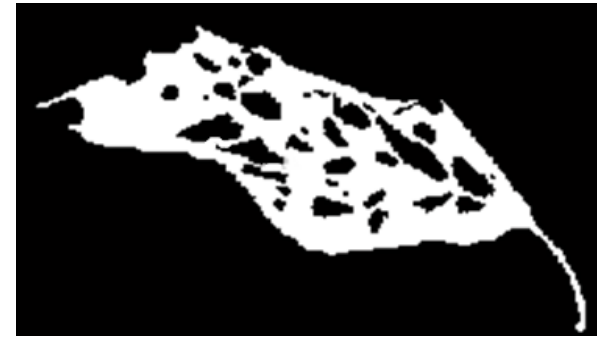

(d) Segmentation of the improved model Figure 2. Experimental Results of a Withering Leaf

By visually observing the results in Figure 1 and Figure 2, we reach the conclusion that the improved model performs better in extracting leaf images than the classical CV model. However, according to the second standard, we need to prove it by giving the exact value of running time of algorithm, in seconds.

Table 1. Results between the Improved Model and C-V Model

\begin{tabular}{ccccc}
\hline & \multicolumn{2}{c}{ Iteration times } & \multicolumn{2}{c}{ Running time } \\
& Figure 1 & Figure 2 & Figure 1 & Figure 2 \\
\hline C-V model & 400 & 1100 & 6.537893 & 23.998653 \\
The improved model & 250 & 700 & 4.161764 & 14.677784 \\
& & & & \\
\hline
\end{tabular}

It can be seen from Table 1 that the improved model's iteration times and running time are less than C-V model's. By numerical analysis, the improved active contours model proposed by this paper is more than 1.5 times faster to $\mathrm{C}-\mathrm{V}$ model, which proves the calculation speed has been improved compared with classical C-V model. Our model is more suitable for the extraction of leaf margin from given images to construct the leaf organs database for the virtual plant reconstruction.

\section{Conclusion}

To the two obvious shortcomings of extraction of leaf margin images of $\mathrm{C}-\mathrm{V}$ model, we combine local statistic information with global information and optimize the defect that SDF needs to be reconstructed partially so that the energy function is improved.

The experiment shows, in the process of leaf margin image extraction, that the improved model is better to overcome the defect that the effect of active contours is not so good when the inside and outside of target edge is homogeneous region. It can extract leaf image more accurately, especially it can keep minutiae features of leaves with special shapes. Meanwhile, it improves the iteration rate and reduces program running time of algorithm. The author experimented with some leaves with various shapes and got the same result as the paper's conclusion.

\section{Acknowledgements}

Fund Project: This paper is supported by the Natural Science Foundation of Heilongiiang Province of China (C201337), Special Funds for Scientific and Technological Innovation Talents of Harbin (2014RFQXJ127), the Fundamental Research Funds for the Central Universities (2572014CB14), and National Natural Science Foundation of China (31470714). 


\section{References}

[1] Y. Li, Q. Zhu, Y. Cao and C. Wang, Editors, "A Leaf Vein Extraction Method based on Snakes Technique", Proceedings of International Conference on Neural Networks and Brain, (2005) October 13-15, Beijing, China.

[2] R. Mesh and P. Prusinkiewicz, Editors, "Visual Models of Plants Interacting with their Environment", In Computer Graphics Proceedings, Annual Conference Series, ACM SIGGRAPH, (1996), NY, USA.

[3] J. Weber and J. Penn, Editors, "Creation and Rendering of Realistic Trees", I Computer Graphics (SIGGRAPH '95 Proceedings), ACM SIGGRAPH, (1995) August, NY, USA.

[4] P. Prusinkiewicz, "Modeling and Visualization of Biological Structures", Proceedings of Graphics Interface '93, (1993) May 19-21, Toronto, Canada.

[5] M. Kass, A. Witkin and D. Terzopoulos, "Snakes: Active Contour Models", International Journal of Computer Vision, vol. 1, no. 321, (1988).

[6] Y. Xiang, A. Chung and J. Ye, "An Active Contour Model for Image Segmentation based on Elastic Interaction", J. Computational Physics, vol. 219, no. 455, (2006).

[7] H. K. Park and M. J. Chung, "External force of Snake: Virtual Electric Field", J. Electronics Letters, vol. 38 , no. 1500 , (2002).

[8] C. Xu and J. L. Prince, "Snakes, Shapes, and Gradient Vector Flow", IEEE Transactions on Image Processing, vol. 7, no. 359, (1998).

[9] L. D. Cohen and I. Cohen, "Finite-element Methods for Active Contour Models and Balloons for 2-D and 3-D Images", IEEE Transactions on Pattern Analysis and Machine Intelligence, vol. 15, no. 1131, (1993).

[10] R. Malladi, J. A. Sethian and B. C. Vemuri, "Shape Modeling with Front Propagation: A Level Set Approach", IEEE Transactions on Pattern Analysis and Machine Intelligence, vol. 17, no. 158, (1995).

[11] R. Fedkiw and S. Osher, "Level Set Methods and Dynamic Implicit Surfaces", J. Surfaces, vol. 44, no. 77, (2002).

[12] S. Osher and J. A. Sethian, "Fronts Propagating with Curvature-dependent Speed: Algorithms Based on Hamilton-Jacobi Formulations", J. Computational Physics, vol. 79, no. 12, (1988).

[13] D. Mumford and J. Shah, "Optimal Approximations by Piecewise Smooth Functions and Associated Variational Problems", J. Communications on Pure and Applied Mathematics, vol. 42, no. 577, (1989).

[14] T. F. Chan and L. A. Vese, "Active Contours Without Edges", IEEE Transactions on Image Processing, vol. 10, no. 266, (2001).

[15] J. Li, X. Yang and L. Yang, "Mumford-Shah Model based Critical Subset Segmentation from Navigation reference images", J. Act a Automatica Sinica, vol. 30, no. 45, (2004).

[16] S. H. Lee and J. K. Seo, "Level set-based bimodal segmentation with stationary global minimum", IEEE Transactions on Image Processing, vol. 15, no. 2843, (2006).

[17] T. Brox and D. Cremers, Editor, "On the Statistical Interpretation of the Piecewise Smooth Mumford-Shah Functional", Scale Space and Variational Methods in Computer Vision, Berlin Heidelberg, (2007), pp. 203-213.

[18] T. Brox and D. Cremers, "On local Region Models and a Statistical Interpretation of the Piecewise Smooth Mumford-Shah Functional", International Journal of Computer Vision, vol. 84, no. 184, (2009).

[19] T. Brox and J. Weickert, "Level Set Segmentation with Multiple Regions", IEEE Transactions on Image Processing, vol. 15, no. 3213, (2006).

[20] T. Chan and W. Zhu, Editors, "Level Set Based Shape Prior Segmentation", Computer Society Conference on Computer Vision and Pattern Recognition (CVPR), IEEE, (2005) June 20-26, CA, USA.

[21] R. Fletcher and M. J. D Powell, “A Rapidly Convergent Descent Method for Minimization”, The Computer Journal, vol. 6, no. 163, (1963).

[22] Jr. A. Yezzi, A. Tsai and A. Willsky, "A Fully Global Approach to Image Segmentation via Coupled Curve Evolution Equations", Journal of Visual Communication and Image Representation, vol. 13, no. 195, (2002).

[23] C. Li, C. Xu, C. Gui and M. D. Fox, Editors, "Level Set Evolution without Re-initialization: a New Variational Formulation", Computer Society Conference on Computer Vision and Pattern Recognition (CVPR), IEEE, (2005) June 20-26, CA, USA.

[24] R. Ksantini, F. Shariat and B. Boufama, "An Efficient and Fast Active Contour Model for Salient Object Detection", Canadian Conference on Computer and Robot Vision (CRV), IEEE, (2009) May 25-27, Kelowna, Canadian.

[25] A. Gelas, O. Bernard, D. Friboulet and R. Prost, "Compactly Supported Radial Basis Functions Based Collocation Method for Level-set Evolution in Image Segmentation", IEEE Transactions on Image Processing, vol. 16, no. 1873, (2007). 
International Journal of $u-$ and e- Service, Science and Technology Vol.8, No. 6 (2015) 